Article

\title{
Personalized hybrid educational recommender system using matrix factorization with user and item information
}

\author{
Paula Andrea Rodriguez-Marin ${ }^{1,2,+}{ }^{\mathbb{C}}$, Nestor Dario Duque-Mendez ${ }^{3}(\mathbb{0}$, Demetrio Arturo \\ Ovalle-Carranza $^{3}(1)$ and Juan David Martinez-Vargas ${ }^{1}$ (1) \\ 1 MIRP Lab-Parque i, Instituto Tecnológico Metropolitano (ITM), Medellín-Colombia; \\ paularodriguez,juanmartinez@itm.edu.co \\ 2 Universidad Nacional de Colombia, sede Medellín; \\ 3 Universidad Nacional de Colombia, sede Manizales; nduquem@unal.edu.co \\ * Correspondence: paularodriguez@itm.edu.co; Tel.: +5734600727 ext 5513
}

\begin{abstract}
One of the main challenges for autonomous learning in virtual environments is finding the right material that fits students' needs and supports their learning process. Personalized recommender systems partially solve this problem by suggesting online educational resources to students based on their preferences. However, in educational environments (which need a proper characterization of both users and educational resources), most existing recommendation algorithms either fail to include all the available information or use hybrid processes that do not exploit possible relationships between users and item features. This article presents a personalized recommender system for educational resources aimed at combining user and item information into a single mathematical model based on matrix factorization. As a result, estimated latent factors can provide insight into possible interactions between users and item features, improving the quality of the information retrieval process. We validated the proposed model on a real dataset that contains the ratings assigned by students from Universidad Nacional de Colombia and Universidade Feevale to educational resources in the Colombian Federation of Learning Object Repositories (FROAC in Spanish). User characterization included learning style and educational level, whereas item characterization (obtained from the objects' metadata), included interactivity level, aggregation level and type, and resource format. These results, compared to those obtained when not all the available information is included, show that our method can improve the recommendation process.
\end{abstract}

Keywords: Hybrid educational recommender system, learning objects, matrix factorization, personalization

\section{Introduction}

Currently, due to the widespread availability of online learning resources and virtual learning environments, the systems and technologies in such field are becoming increasingly popular because they can improve educational access for anyone anywhere in the world [1]. An Open Educational Resource (OER) can be defined as a type of material with an educational purpose and aim, whose information is digitalized and available on the Internet [2-4]. An important feature of OERs is their descriptive metadata, which includes information characterizing the content of the resources, e.g., title, keywords, and educational use [5]. Such metadata enable and facilitate the identification, search, retrieval, and reuse of said resources. OERs and their corresponding metadata are stored in repositories that can organize, centralize, and make them available and visible so that students discover adequate materials in their searches [6]. 
In spite of their availability, advanced searches are difficult, even for senior students, due to the definition of the criteria and selection of the search string [7]. For instance, a study concluded that students should spend more than one hour to find an adequate resource, which is frustrating for them [8]. To deal with this problem, repositories are implementing different measures to improve search processes; some of them use ranking metrics, quality control mechanisms, or Educational Recommender Systems (ERSs).

ERSs are tools that offer personalized suggestions to users by predicting, for example, their level of satisfaction with a given resource [9-11]. Such systems are based on preferences, likes, moods, learning styles, and all the variables that enable the characterization of the user and the retrieval process [12]. Consequently, ERSs personalize recommendations so that they are adapted to each user's characteristics [1]. Moreover, recommendations are often based on previous interactions between users and items because past interests and activities are generally good indicators of future options [13] [14]. ERSs should allow feedback processes and implement mechanisms to obtain a substantial amount of information about users. As a result, the main objective of ERSs in virtual learning environments is to provide students with search results that support their autonomous learning process $[4,7]$.

Existing Recommendation Systems (RSs) can be roughly classified into content-based, demographic-based, and collaborative filtering-based. Content-based RSs make recommendations according to inferences about users' needs and preferences depending on their browsing history [15].Demographic recommender systems use information about the demographic factors of the population under study to find similarities between users and thus provide recommendations [16]. In contrast, collaborative filtering aims to find characteristics users share (through previous ratings) to retrieve items similar users like[17].

Collaborative filtering is commonly categorized into memory-based, model-based, and hybrid methods [18]. Memory-based methods use similarities between users or items based on previous ratings to generate recommendations. Although they are easy to implement, and the provided recommendation is usually easy to explain, memory-based methods do not work well with sparse rating matrices. To overcome such issue, model-based methods characterize both items and users employing latent factors that are inferred from rating patterns. Thus, said models aim at learning from the available data, generalizing behaviors and considering computational complexity [19]. Finally, hybrid methods combine several techniques of RSs to maximize results, making the most of the advantages of each technique and reducing their problems. The more available information sources, the more flexible the system is regarding the use of different types of RSs to find the same items[20].

Among all the collaborative filtering techniques, matrix factorization is the most popular option due to its capability to deal with large datasets [21-23] and become hybrid by combining explicit and implicit attributes of users and items [24]. Matrix factorization methods for RSs characterize both resources and users using a set of characteristics defined in a vectorial manner [18,25,26]. In [19], the authors introduce the algorithms that won the Netflix Prize Competition. They maintain that matrix factorization models/algorithms outperform classical nearest-neighbor techniques in generating item recommendations. Moreover, in [18], the authors propose a nonnegative matrix factorization model with regularization and weighing employing graphs to perform collaborative filtering. They created two graphs: the first one contains users' demographic information (occupation); and the second, movie genres and their relationships. They concluded that such type of method improves the accuracy of the recommendations. Likewise, in [23], the authors tried to improve collaborative filtering by using matrix factorization and exponential random graphs and incorporating social networks. By contrast, in [21], the authors proposed a collaborative filtering method using negative and positive factorization matrices and Pearson Correlation Coefficient (PCC) to improve the quality of user experience. Their process adds restrictions and adjusts the similarity values of users employing only user ratings for the elements to be recommended. Finally, in [26], the authors used matrix factorization algorithms to recommend people. They model attitudes and feelings between individuals in matrices to establish the degree of correlation among them and thus make recommendations. 
Although the above-mentioned recommendation methods achieve good results, most of them complete retrieval tasks by combining several aspects of different types of techniques to obtain the best of both worlds, namely, content- and user-based RSs [27]. Consequently, a hybrid integrated model should include, in a single mathematical model, in addition to the ratings, the available user characteristics and item metadata in the personalized recommendation process. This is particularly true in educational environments, where the system has access to user information (e.g., learning style and educational level) and item information (e.g., interactivity level and semantic density).

This work presents a hybrid personalized recommender system for educational materials that combines recommendation techniques based on collaborative filtering, content, and demographics into a latent factor model. Thus, the proposed system considers the available user information as well as item metadata and infers the existing relationship between those characteristics, improving the recommendations offered to each student.

This article is organized as follows. Section 2 presents the matrix factorization methods for recommender systems and introduces the proposed hybrid and personalized model. Section 3 describes the real dataset along with user and item characteristics. Section 4 details the validation of the proposed system and compares it with state-of-the-art methods. Finally, Section 5 draws conclusions and proposes future work.

\section{Materials and Methods}

\subsection{Matrix factorization methods for recommender systems}

Matrix factorization methods for recommender systems are based on latent factor models, where items as well as users are characterized using vectors of factors inferred through ratings. Consider a set of items, where ${ }_{i} \in \mathfrak{R}^{N \times 1}$ represents a set of $N$ latent factors or characteristics that describe the $i-$ th item. Additionally, each user is associated with a vector $\mathbf{x}_{u} \in \mathfrak{R}^{N \times 1}$. Thus, the elements of ${ }_{i}$ measure how much each factor represents item $i$ (positive or negative), while the elements of $\mathbf{x}_{u}$ measure the degree of interest the user $u$ exhibits in each one of the factors that characterize the items (positive or negative). Furthermore, the dot product of $\mathbf{x}_{u}$ and ${ }^{`}$

$$
\widehat{y}_{u i}=\mathbf{x}_{u}^{\top}{ }^{i},
$$

captures the interaction between user $u$ and item $i$ and can be seen as an approximation of the rating that such user assigns to said item $\widehat{y}_{u i} \in \mathfrak{R}$. Therefore, the challenge for matrix factorization methods is to calculate, from the set of given ratings, the vectors of factors $\mathbf{x}_{u}$ and ${ }_{i}{ }_{i}$ for all the users and all the items. As a result, after the recommender system completes the factorization, it can estimate the ratings users will assign to any item using eq. (1).

The vectors of item factors can be grouped in matrix $\Theta \in \mathfrak{R}^{I \times N}$, where the $i$-th row of the matrix represents the factors of the item, and $I \in \mathfrak{Z}$ denotes the number of items. Likewise, user factors can be grouped in matrix $\mathbf{X} \in \mathfrak{R}^{U \times Z}$, where the $u$-th row represents the factors of user, and $U \in \mathfrak{N}$ denotes the number of users. Moreover, the ratings users have assigned to the items can be stored in matrix $\mathbf{Y} \in \mathfrak{R}^{U \times I}$. Because not all users have evaluated all the items, a matrix $R \in[0,1]^{U \times I}$ is created, where $r_{u i}=1$ ( $u$-th row, $i$-th column of $\mathbf{R}$ ) if user $u$ has already rated item $i$, and $r_{u i}=0$ in the oppossite case. Thus, to estimate factor matrices, the recommender system minimizes the regularized mean squared error using known ratings

$$
\begin{aligned}
L(\mathbf{X}, \boldsymbol{\Theta}) & =\frac{1}{2}\left\|\left(\mathbf{Y}-\mathbf{X} \boldsymbol{\Theta}^{\top}\right) \odot \mathbf{R}\right\|_{F}^{2}+\frac{\lambda}{2}\left(\|\mathbf{X}\|_{F}^{2}+\|\boldsymbol{\Theta}\|_{F}^{2}\right) \\
\{\widehat{\mathbf{X}}, \widehat{\boldsymbol{\Theta}}\} & =\underset{\mathbf{X}, \boldsymbol{\Theta}}{\operatorname{argmin}}\{L(\mathbf{X}, \boldsymbol{\Theta})\},
\end{aligned}
$$


where $\lambda \in \mathfrak{R}^{+}$is a regularization parameter; $\|\cdot\|_{F}$ the Frobenius norm; and $\odot$, the Hadamard product. Under this formulation, the system learns the model by adjusting the previously observed ratings. Additionally, since the objective is to generalize prior ratings so that they enable the prediction of yet unassigned ratings, a regularization term is used to avoid overtraining.

Matrices $\mathbf{X}$ and $\boldsymbol{\Theta}$ are estimated using Gradient Descent, in the form

$$
\begin{aligned}
& \mathbf{X}_{j}=\mathbf{X}_{j-1}-\gamma \frac{\partial}{\partial \mathbf{X}} L(\mathbf{X}, \boldsymbol{\Theta}) \\
& \boldsymbol{\Theta}_{j}=\mathbf{\Theta}_{j-1}-\gamma \frac{\partial}{\partial \boldsymbol{\Theta}} L(\mathbf{X}, \boldsymbol{\Theta})
\end{aligned}
$$

where $\gamma \in \mathfrak{R}^{+}$is the learning rate, matrices $\mathbf{X}_{j}, \boldsymbol{\Theta}_{j}$ denote the $j$-th iteration of the optimization algorithm, and the derivatives are defined as

$$
\begin{aligned}
& \frac{\partial L(\mathbf{X}, \boldsymbol{\Theta})}{\partial \mathbf{X}}=-(\mathbf{R} \odot \mathbf{Y}) \boldsymbol{\Theta}+\mathbf{R} \odot\left(\mathbf{X} \boldsymbol{\Theta}^{\top}\right) \boldsymbol{\Theta}+\lambda \mathbf{X} \\
& \frac{\partial L(\mathbf{X}, \boldsymbol{\Theta})}{\partial \boldsymbol{\Theta}}=-(\mathbf{R} \odot \mathbf{Y})^{\top} \mathbf{X}+\left(\mathbf{R} \odot\left(\mathbf{X} \boldsymbol{\Theta}^{\top}\right)\right)^{\top} \mathbf{X}+\lambda \boldsymbol{\Theta}
\end{aligned}
$$

\subsection{Hybrid recommender system using matrix factorization along with user and item information}

In addition to ratings, recommender systems may include different sources of information about users and/or items in order to improve predictions. Hence, search history or learning style characterization can be used to describe user trends and create a matrix of factors $\mathbf{X}$. Therefore, the unknowns in eq. (2) will only be the factors of users $\Theta$, and they can be estimated using eqs. (3b) and (4b). Therefore, $\theta_{i f}$ (row $i$, column $f$ of $\boldsymbol{\Theta}$ ) will indicate how much of characteristic $f$ that describes all the users appears in item $i$. If a characterization of the items $\boldsymbol{\Theta}$ is available (e.g., describing the level of interactivity or how much it contributes to each learning style), the unknowns in eq. (2) will be the factors of users $\mathbf{X}$, and they can be estimated using eqs. (3a) and (4a). As a result, $x_{u f}$ (row $u$, column $f$ of $\mathbf{X}$ ) will indicate how important characteristic $f$ that describes the items is to user $u$.

However, when descriptions of the users as well as the items (i.e., matrices $\mathbf{X}$ and $\boldsymbol{\Theta}$ ) are available, the formulation of eq. (2) can only be applied i there is a direct correspondence between the characterization of items and that of users, which includes (i) an equal number of characteristics and (ii) the same characterization for users and items. Therefore, since in few real cases such characterization correspondence can be obtained, this work proposes a model that allows the utilization of user and item descriptions. For that purpose, users are assumed to be described with a number $N_{u}$ of characteristics, which produces matrix $\mathbf{X} \in \mathcal{R}^{U \times N_{u}}$. In turn, items are described with a number $N_{i}$ of characteristics, which produces matrix $\Theta \in \mathcal{R}^{I \times N_{i}}$. Thus, eq. (2) can be rewritten including a matrix of factors $\Sigma \in \mathcal{R}^{N_{u} \times N_{i}}$, as follows

$$
\begin{aligned}
L(\boldsymbol{\Sigma}) & =\frac{1}{2}\left\|\left(\mathbf{Y}-\mathbf{X} \boldsymbol{\Sigma} \boldsymbol{\Theta}^{\top}\right) \odot \mathbf{R}\right\|_{F}^{2}+\frac{\lambda}{2}\|\boldsymbol{\Sigma}\|_{F}^{2} \\
\widehat{\boldsymbol{\Sigma}} & =\underset{\boldsymbol{\Sigma}}{\operatorname{argmin}}\{L(\boldsymbol{\Sigma})\},
\end{aligned}
$$

The derivative of this cost function with respect to parameters $\Sigma$ is defined as

$$
\frac{\partial}{\partial \boldsymbol{\Sigma}} L(\boldsymbol{\Sigma})=-\frac{1}{2} \mathbf{X}^{\top}(\mathbf{Y} \odot \mathbf{R}) \boldsymbol{\Theta}+\mathbf{X}^{\top}\left(\left(\mathbf{X} \boldsymbol{\Sigma} \boldsymbol{\Theta}^{\top}\right) \odot \mathbf{R}\right)+\lambda \boldsymbol{\Sigma} .
$$

Thus, we use gradient descent to optimize the model parameters, as follows

$$
\Sigma_{j}=\Sigma_{j-1}-\gamma \frac{\partial}{\partial \Sigma} L(\Sigma),
$$


where $\gamma \in \mathfrak{R}^{+}$is the learning rate.

According to eq. (1), once matrix $\Sigma$ has been obtained, the estimation of the rating that user $u$ will assign to item $i$ is given by:

$$
\widehat{y}_{u i}=\sum_{n_{i}=1}^{N_{i}} \mathbf{x}_{u} \mathbf{e}_{n i} \theta_{n_{i}, i}
$$

where $\mathbf{x}_{u} \in \mathcal{R}^{1 \times N_{u}}$ are the characteristics of user $u$; $\boldsymbol{\aleph}_{n i} \in \mathcal{R}^{1 \times N_{u}}$, the relationships between the characteristics of user $u$ and object $i$; and $\theta_{n_{i}, i}$ the $n_{i}$-th characteristic of item $i$.

\subsection{The cold start problem of new users}

Oftentimes, recommender systems need to deal with the cold start problem when a new user has rated a few or none of the items, thus making the prediction task more difficult. One way to overcome this problem is to use the user factors $\mathbf{X}$ to find similarities between the new user and the existing ones in the database. As a result, the ratings of the new user can be obtained as the average of the ratings of the users that are most similar to said user. The Pearson Correlation Coefficient (PCC) is employed as a measure of similarity between user $u$ and user $u^{\prime}$, defined as

$$
\operatorname{Sim}\left(u, u^{\prime}\right)=\frac{\sum_{n_{u}=1}^{N_{u}}\left(x_{u, n_{u}}-\bar{x}_{u}\right)\left(x_{u^{\prime}, n_{u}}-\bar{x}_{u^{\prime}}\right)}{\sqrt{\sum_{n_{u}=1}^{N_{u}}\left(x_{u, n_{u}}-\bar{x}_{u}\right)^{2}} \sqrt{\sum_{n_{u}=1}^{N_{u}}\left(x_{u^{\prime}, n_{u}}-\bar{x}_{u^{\prime}}\right)^{2}}},
$$

where $x_{u, n_{u}} \in \mathcal{R}$ is the $n_{u}$-th characteristic of user $u ; \bar{x}_{u} \in \mathcal{R}$ denotes the average of the characteristics of user $u$; and $\operatorname{Sim}\left(u, u^{\prime}\right) \in[-1,1]$. Hence, a subgroup of users whose similarity exceeds a threshold with respect to the new user is selected. The initial estimation of the ratings assigned by the new user are calculated as the average of the ratings that the most similar users have assigned.

This approach seeks to use the greatest possible amount of item and user information to deliver relevant recommendations based on the idea that the more is known about the user and the item, the better the results. Likewise, the objective is to produce a model that works when characteristics are known or unknown, as in the case of a new user. If a new user in the recommender system has rated a few or none of the items, a similarity measure is then used through PCC to find users that have similar characterizations. Thus, the initial estimation of the ratings assigned by the new user are calculated as the average of the ratings that the most similar users have assigned.

\section{Validation}

The hybrid recommender system for educational resources proposed in this work comprises three steps: (i) characterizing users (students) and items (learning objects), (ii) estimating the unknowns in the model, and (iii) completing the score matrices to submit the recommendation. To evaluate the performance of the proposed model, the recommendations were assessed using a real educational dataset that contains user information, metadata of the OERs, and the ratings users assigned to such resources. This dataset stores a selection of educational resources from the Colombian Federation of Learning Object Repositories (FROAC in Spanish) available at http:/ / froac.manizales.unal.edu. co/froacn/. Figure 1 illustrates, step by step, the proposed model (blue lines) compared to other alternatives. The lines indicate the information (user features, item features, and scores) that is fed into each model. 


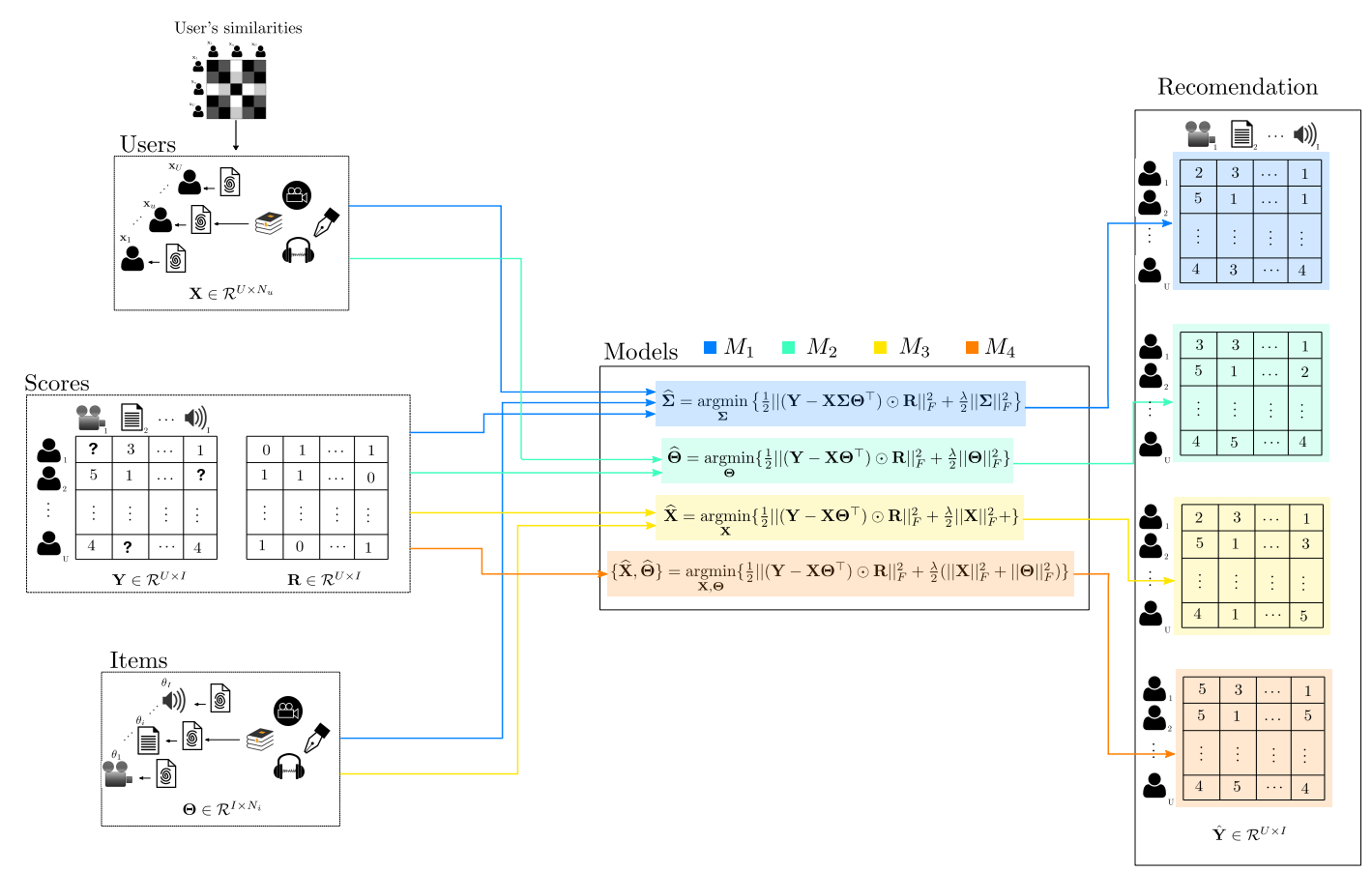

Figure 1. Illustration of the proposed recommender system of educational resources. The left side shows all the available information sources (user features, item features, and scores). Lines of different colors indicate the information that is fed into each model under testing. Finally, one recommendation per model is obtained on the right.

Each step mentioned above and the database are detailed below.

\subsection{Data-set}

The educational data set is composed of user information, metadata of the OERs, and the ratings assigned by the users to the items. The selection of the characteristics was completed considering the previous experience of the group and highlighting learning style as the most relevant factor [12][28].

\section{User characterization:}

A total of $U=56$ users participated in the study. Users' information excludes personal data to respect students' privacy. Users were identified and the distribution of learning styles was obtained from the answers to the proposed test, where students were classified adopting the VARK model [29] and Felder-Silverman Learning Style Model [30] regarding information processing, i.e., global/sequential. The user information collected in this study is detailed below:

- User ID: Unique user identification for each user.

- visual: They prefer the use of symbolism and different formats, fonts, and colors to highlight important points.

- auditory: They prefer spoken information that can be heard, and posing questions is an important part of their learning strategies.

- reading/writing: They use print words as the most important way to transmit and receive information.

- kinesthetic: They use their own experience and real things, even when presented in images or screens.

- global:They have holistic systemic minds that learn quickly.

- Sequential: They benefit from linear, organized processing and learn better taking small incremental steps. 
According to the VARK model, the participants in this study were categorized into visual $(12 \%)$, auditory $(18 \%)$, reading/writing $(20 \%)$, and kinesthetic $(50 \%)$. Further, according to the Felder-Silverman learning style model, students were classified into global (30\%) and sequential $(70 \%)$.

Item characterization:

A total of educational resources were included in this study; their metadata describe their contribution to each learning style. The definition of each type of metadata was taken from the IEEE Standard for Learning Object Metadata (IEEE-LOM) [31]. Item information is explained below:

- Item ID: Unique identification for each resource.

- Aggregation Level: Aggregation level of the resource with respect to its granularity.

- Structure: Underlying organizational structure of the educational resource.

- Interactivity Type: Predominant learning style supported by the resource, i.e., active, expository or mixed learning.

- Interactivity Level: Degree of interactivity that characterizes the educational resource. In this context, interactivity refers to the degree to which the student can influence the appearance or behavior of the resource.

- Semantic Density: Degree of conciseness of the OER. The semantic density of an object can be estimated in terms of its size, field or-in the case of self-timed resources such as audio and video-duration. The semantic density of an educational resource is independent of its difficulty.

- Visual: Contribution of the resource for a student with a visual learning style.

- Auditory: Contribution of the resource for a student with an auditory learning style.

- Reading: Contribution of the resource for a student with a reading learning style.

- Kinesthetic: Contribution of the resource for a student with a kinesthetic learning style.

Scores:

This file stores all the ratings assigned by the users to the items in five categories, as well as user and item identifications.

- User ID: Identification of the user who completed the evaluation.

- Item ID: Identification of the resource that was rated.

- R1: Overall rating.

- R2: Contribution to learning.

- R3: Design.

- R4: Content quality.

- R5: Likelihood of recommending this resource.

The information was collected by randomly assigning OERs to students and asking them to evaluate each of them. Figure 2 presents the interface that was used to collect such evaluations. 


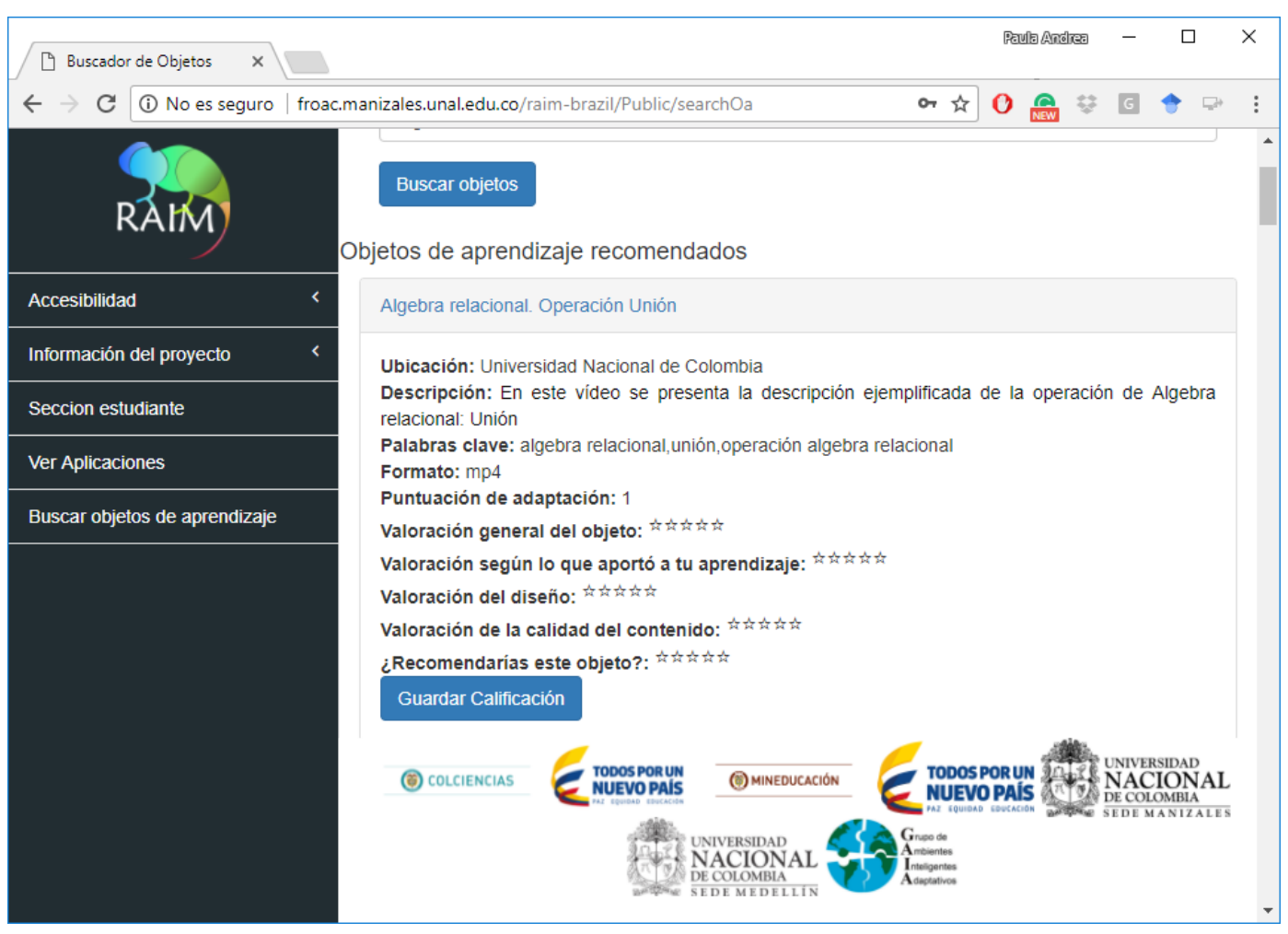

Figure 2. Information collection interface for the data set.

At the end, the data-set included 400 evaluations of 152 OERs by 56 users (students from Feevale University in Brazil and the National University of Colombia). The proposed recommender system was applied using those data. Hence, the users file was matrix $\mathbf{X} \in \mathcal{R}^{56 \times 6}$, and resources were $\boldsymbol{\Theta} \in \mathcal{R}^{152 \times 9}$. Moreover, five evaluation matrices $\mathbf{Y}_{r} \in \mathcal{R}^{56 \times 152}, r=1, \ldots, 5$, which correspond to each rating, were individually tested.

\subsection{Proposed model and models for comparison}

When all the information is available (users, items, and evaluations) different matrix factorization algorithms can be applied to estimate the missing ratings. Thus, according to the available information, parameters of the recommendation model based on matrix factorization vary as detailed below:

- $\mathbf{M}_{\mathbf{1}}$ The proposed model uses the information of items $\boldsymbol{\Theta}$ as well as users $\mathbf{X}$, and matrix $\boldsymbol{\Sigma} \in \mathcal{R}^{6 \times 9}$ is inferred, for a total of 54 parameters to be estimated. $\Sigma$ represents the relationship between item and user characteristics.

- $\mathbf{M}_{2}$ The second model uses the characteristics of users $\mathbf{X}$. Therefore, matrix $\boldsymbol{\Theta} \in \mathcal{R}^{152 \times 9}$ is inferred, for a total of 1368 parameters to be estimated.

- $\mathbf{M}_{3}$ The third model considers only item characteristics, i.e., matrix $\Theta$. Subsequently, $\mathbf{X} \in \mathcal{R}^{56 \times 6}$ is estimated, with 336 parameters.

- $\mathbf{M}_{4}$ The fourth model does not take into account resource or user characteristics. Consequently, $\boldsymbol{\Theta} \in \mathcal{R}^{152 \times 9}$ and $\mathbf{X} \in \mathcal{R}^{56 \times 6}$ must be estimated, for a total of 1704 parameters.

For models $M_{1}$ and $M_{2}$, which take into account user characteristics, each feature (learning style) was scaled from 0 to 10 .

For models $M_{1}$ and $M_{3}$, the object metadata of each item, discarding the itemID, were scaled from 0 to 10 .

Finally, for all models, scores $R_{1}$ to $R_{5}$ where scaled from 0 to 5 .

Table 1 presents a summary comparing the models along with their cost functions, the matrices to be estimated, and the number of parameters. 
Table 1. Compared recommendation systems along with their unknows and number of parameters.

\begin{tabular}{c|c|c|c} 
Model & Cost function & Unknowns & Parameters \\
\hline$M_{1}$ & $\frac{1}{2}\left\|\left(\mathbf{Y}-\mathbf{X} \boldsymbol{\Sigma} \boldsymbol{\Theta}^{\top}\right) \odot \mathbf{R}\right\|_{F}^{2}+\frac{\lambda}{2}\|\mathbf{\Sigma}\|_{F}^{2}$ & $\boldsymbol{\Sigma}$ & 54 \\
$M_{2}$ & $\frac{1}{2}\left\|\left(\mathbf{Y}-\mathbf{X} \boldsymbol{\Theta}^{\top}\right) \odot \mathbf{R}\right\|_{F}^{2}+\frac{\lambda}{2}\|\boldsymbol{\Theta}\|_{F}^{2}$ & $\boldsymbol{\Theta}$ & 1368 \\
$M_{3}$ & $\frac{1}{2}\left\|\left(\mathbf{Y}-\mathbf{X} \boldsymbol{\Theta}^{\top}\right) \odot \mathbf{R}\right\|_{F}^{2}+\frac{\lambda}{2}\|\mathbf{X}\|_{F}^{2}$ & $\mathbf{X}$ & 336 \\
$M_{4}$ & $\frac{1}{2}\left\|\left(\mathbf{Y}-\mathbf{X} \boldsymbol{\Theta}^{\top}\right) \odot \mathbf{R}\right\|_{F}^{2}+\frac{\lambda}{2}\left(\|\mathbf{X}\|_{F}^{2}+\|\boldsymbol{\Theta}\|_{F}^{2}\right)$ & $\mathbf{\Sigma}, \boldsymbol{\Theta}$ & 1704
\end{tabular}

\subsection{Evaluation of the compared models}

Several measurements have been employed to evaluate the quality of predictions (also called performance) of recommender systems [32]. The most commonly used measure is the Mean Squared Error (MSE), which can be used to quantify the deviation of forecasted recommendations from real values. The lower the MSE, the better the prediction made by the system. The MSE is defined as

$$
M S E=\frac{1}{N} \sum_{i=1}^{N}\left(x_{i}-\hat{x}_{i}\right)^{2} .
$$

User ratings of items are commonly divided into a training set, used to learn, and a test set, used to evaluate the quality of the predictions by measuring the MSE between the actual rating and the prediction [33].

Tests were also conducted considering a lack of user ratings (the cold start problem) and employing similarity in user characteristics to find the resources that a user may like without having assigned previous ratings. It should be mentioned that all the values underwent a normalization process so that their range was between 0 and 5 .

\subsection{Experimentation and validations}

Different experiments were conducted using the MSE to compare the proposed model with the three other models considered in this work; in this case, the lower the MSE, the better the resulting recommendation.

The first experiment implemented a cross-validation methodology with 10 partitions using all the available ratings. For that purpose, the 400 ratings were randomly divided into 10 groups with approximately 40 ratings each. Subsequently, 9 out of the 10 groups were used to train the algorithm and estimate the unknowns, while the remaining group was used for the validation. This experiment was repeated until each group was employed for the validation.

The second experiment considered the cold start problem of new users. For that purpose, the ratings of 55 out of the 56 users were the training data, while the ratings of the remaining user were employed for the validation. In order to calculate the initial value of the ratings of the validation user, the PCC between the characteristics of said user and those of all the users in the training set was obtained. Afterward, user ratings with a PCC over 0.7 were averaged. This process was repeated until each user was employed for the validation.

In addition to the 5 types of ratings initially considered in this work (i.e., overall rating, $R_{1}$; contribution to learning, $R_{2}$; design, $R_{3}$; content quality, R4; and likelihood of recommending the resource, $R_{5}$ ), three other ratings were created. Such ratings were weighted in order to include even more information about users in the model and make it more general. First, the ratings were weighted as if all of them were equally important in the system; this adapted rating was named $R_{6}$. Afterward, an analysis by the research team concluded that the most important ratings should be those regarding the contribution to the learning process and the quality of the content in the resource. As a result, a more significant weight was assigned to those two aspects, which produced $R_{7}$. At the end, it was decided that the first rating (i.e., the overall rating of the resource) is also an important value to be 
considered in the model; thus, $R_{8}$ was created. Equation 10 presents the weights assigned to the 5 ratings included in the model.

$$
\begin{aligned}
& R_{6}=0.2 R_{1}+0.2 R_{2}+0.2 R_{3}+0.2 R_{4}+0.2 R_{5} \\
& R_{7}=0.1 R_{1}+0.35 R_{2}+0.1 R_{3}+0.35 R_{4}+0.1 R_{5} \\
& R_{8}=0.2 R_{1}+0.3 R_{2}+0.1 R_{3}+0.3 R_{4}+0.1 R_{5}
\end{aligned}
$$

Figures 3(a) and 3(b) are box-and-whisker plots of the MSE obtained in Experiments 1 and 2, respectively. Different values of the regularization parameter were used, namely, $\lambda=$ $\{0.01,0.1,1,10,100\}$, and the best results are reported. In both cases, the simplest model $\left(M_{4}\right)$, which does not include the characteristics of users or items, produced the worst results. Additionally, it can be seen that results improve as more information about users and/or items is included. Moreover, user information exerts a greater influence on the recommendation than item information. Because of that, $M_{2}$ (with user information) outperforms $M_{3}$ (with item information). This difference is more clearly noticeable in Experiment 2, where including item information does not improve the results of the model compared to only considering the ratings available in the training set $\left(M_{4}\right)$. This behavior can be explained by the fact that, in the cold start experiment, user information is also employed to provide an initial estimation of the ratings assigned by the validation user. Finally, the proposed model $\left(M_{1}\right)$, including user as well as item information, obtained the best results overall, which implies better recommendations. 


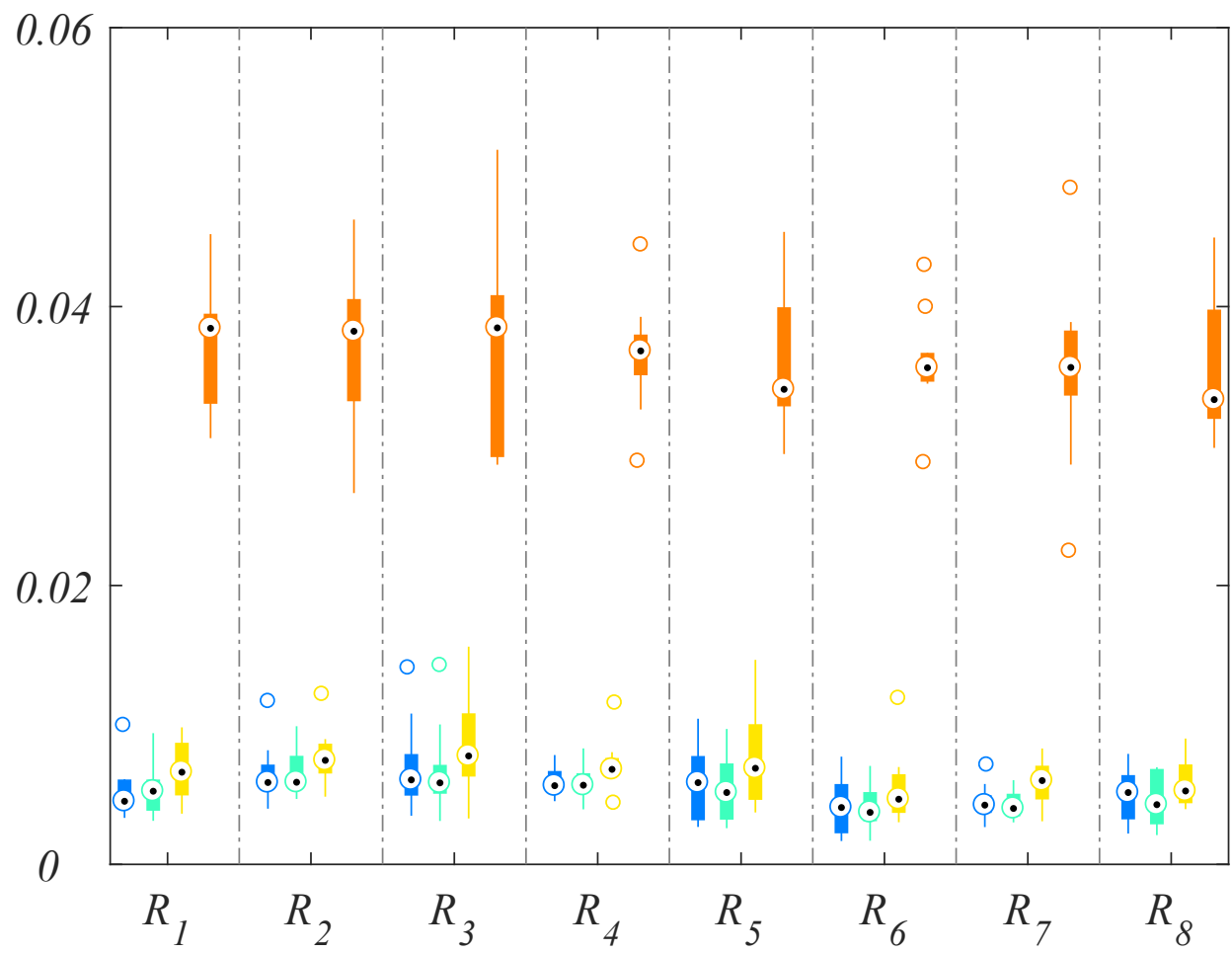

(a) Cross-validation

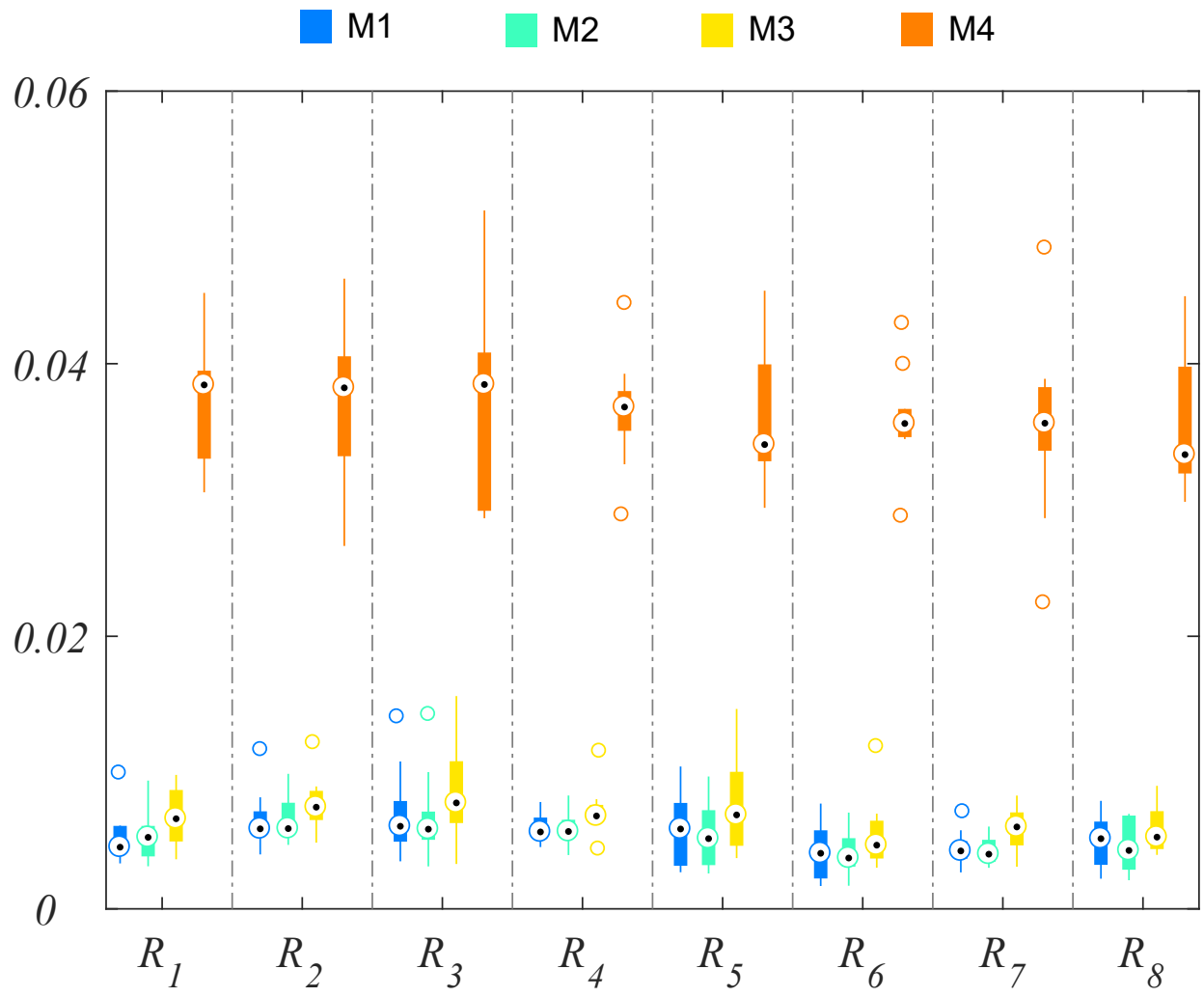

(b) Cold start

Figure 3. Achieved MSE with all the ratings and models for both considered experiments 
In order to demonstrate that the proposed model achieves better results than the models for comparison, their statistical difference was quantified. For that purpose, a paired Student's t-test was employed with a null hypothesis: there are no significant differences between the reference model $\left(M_{1}\right)$ and each model for comparison. In turn, an alternative hypothesis was also tested : the average MSE value of the proposed model is different to that of the models for comparison. Additionally, $\mathrm{t}$-statistic values below 0 indicated that the MSE of $M_{1}$ was lower than that of the model to which it was compared. P-values measured the significance of the difference. P-values under 0.05 ( $5 \%$ of significance) indicated a rejection of the null hypothesis (the alternative hypothesis was accepted). The results of Student's t-test are presented in table 2.

Table 2. Paired Student's t-test comparing the recommendation models. ${ }^{*}$ denotes a significance level $\mathrm{p}=0.05$; and ${ }^{* *}$, a significance level $p=0.01$.

\begin{tabular}{|c|c|c|c|c|c|c|c|}
\hline \multirow{2}{*}{ Rating } & \multirow{2}{*}{ Compared model } & \multicolumn{3}{|c|}{ Cross-validation } & \multicolumn{3}{|c|}{ Cold start } \\
\hline & & h & $\mathbf{t}$ & p & $\mathrm{h}$ & $t$ & p \\
\hline$R_{1}$ & $\begin{array}{l}M_{2} \\
M_{3} \\
M_{4}\end{array}$ & $\begin{array}{l}0 \\
1 \\
1\end{array}$ & $\begin{array}{c}-1.3 \\
-31.029 \\
-18.913 \\
\end{array}$ & $\begin{array}{c}0.22589^{*} \\
0.012664^{* *} \\
1.49 e^{-4^{* *}}\end{array}$ & $\begin{array}{l}1 \\
1 \\
1\end{array}$ & $\begin{array}{l}-42.628 \\
-67.926 \\
-66.956\end{array}$ & $\begin{array}{l}8.15 e^{-1^{* *}} \\
8.95 e^{-5^{* *}} \\
1.29 e^{-4^{* *}}\end{array}$ \\
\hline$R_{2}$ & $\begin{array}{l}M_{2} \\
M_{3} \\
M_{4}\end{array}$ & $\begin{array}{l}0 \\
1 \\
1\end{array}$ & $\begin{array}{c}-2.72 e^{-4} \\
-28.297 \\
-17.737 \\
\end{array}$ & $\begin{array}{c}0.99979^{*} \\
0.019731^{*} \\
2.61 e^{-4^{* *}}\end{array}$ & $\begin{array}{l}1 \\
1 \\
1 \\
\end{array}$ & $\begin{array}{l}-39.325 \\
-63.357 \\
-63.211 \\
\end{array}$ & $\begin{array}{l}2.41 e^{-4^{* *}} \\
4.92 e^{-4^{* *}} \\
5.19 e^{-4^{* *}}\end{array}$ \\
\hline$R_{3}$ & $\begin{array}{l}M_{2} \\
M_{3} \\
M_{4}\end{array}$ & $\begin{array}{l}0 \\
1 \\
1\end{array}$ & $\begin{array}{c}10.206 \\
-3.61 \\
-14.631 \\
\end{array}$ & $\begin{array}{c}0.33408 \\
0.005659^{*} \\
1.4 e^{-7^{* *}}\end{array}$ & $\begin{array}{l}1 \\
1 \\
1\end{array}$ & $\begin{array}{l}-42.289 \\
-68.317 \\
-68.375 \\
\end{array}$ & $\begin{array}{l}9.13 e^{-1^{* *}} \\
7.73 e^{-5^{* *}} \\
7.56 e^{-5^{* *}} \\
\end{array}$ \\
\hline$R_{4}$ & $\begin{array}{l}M_{2} \\
M_{3} \\
M_{4}\end{array}$ & $\begin{array}{l}0 \\
1 \\
1\end{array}$ & $\begin{array}{l}0.41004 \\
-31.229 \\
-243811 \\
\end{array}$ & $\begin{array}{c}0.69137 \\
0.01226^{* *} \\
1.57 e^{-5^{* *}}\end{array}$ & $\begin{array}{l}1 \\
1 \\
1\end{array}$ & $\begin{array}{l}-40.533 \\
-66.433 \\
-66.239 \\
\end{array}$ & $\begin{array}{l}1.62 e^{-4^{* *}} \\
1.56 e^{-4^{* *}} \\
1.68 e^{-4^{* *}}\end{array}$ \\
\hline$R_{5}$ & $\begin{array}{l}M_{2} \\
M_{3} \\
M_{4} \\
\end{array}$ & $\begin{array}{l}1 \\
1 \\
1 \\
\end{array}$ & $\begin{array}{c}25.228 \\
-2.938 \\
-16.215 \\
\end{array}$ & $\begin{array}{c}0.03262 \\
0.01654^{* *} \\
5.72 e^{-4^{* *}}\end{array}$ & $\begin{array}{l}1 \\
1 \\
1\end{array}$ & $\begin{array}{l}-4.021 \\
-66.719 \\
-65.752 \\
\end{array}$ & $\begin{array}{l}1.81 e^{-4^{* *}} \\
1.40 e^{-4^{* *}} \\
2.01 e^{-4^{* *}}\end{array}$ \\
\hline$R_{6}$ & $\begin{array}{l}M_{2} \\
M_{3} \\
M_{4} \\
\end{array}$ & $\begin{array}{l}0 \\
1 \\
1 \\
\end{array}$ & $\begin{array}{l}0.52056 \\
-33.316 \\
-27.771 \\
\end{array}$ & $\begin{array}{c}0.61524 \\
8.77 e^{-3^{* *}} \\
4.94 e^{-6^{* *}} \\
\end{array}$ & $\begin{array}{l}1 \\
1 \\
1 \\
\end{array}$ & $\begin{array}{l}-40.727 \\
-65.752 \\
-66.234 \\
\end{array}$ & $\begin{array}{l}1.53 e^{-4^{* *}} \\
2.09 e^{-5^{* *}} \\
1.68 e^{-4^{* *}}\end{array}$ \\
\hline$R_{7}$ & $\begin{array}{l}M_{2} \\
M_{3} \\
M_{4}\end{array}$ & $\begin{array}{l}0 \\
1 \\
1 \\
\end{array}$ & $\begin{array}{c}0.71407 \\
-56.399 \\
-14.83 \\
\end{array}$ & $\begin{array}{c}0.49328 \\
3.17 e^{-4^{* *}} \\
1.25 e^{-3^{* *}}\end{array}$ & $\begin{array}{l}1 \\
1 \\
1\end{array}$ & $\begin{array}{l}-39.646 \\
-65.345 \\
-65.242 \\
\end{array}$ & $\begin{array}{l}2.17 e^{-4^{* *}} \\
2.35 e^{-5^{* *}} \\
2.44 e^{-4^{* *}}\end{array}$ \\
\hline$R_{8}$ & $\begin{array}{l}M_{2} \\
M_{3} \\
M_{4}\end{array}$ & $\begin{array}{l}0 \\
0 \\
1\end{array}$ & $\begin{array}{c}17.804 \\
-16.176 \\
-20.013\end{array}$ & $\begin{array}{c}0.1087 \\
0.1402^{*} \\
9.03 e^{-6^{* *}}\end{array}$ & $\begin{array}{l}1 \\
1 \\
1\end{array}$ & $\begin{array}{l}-40.144 \\
-64.831 \\
-65.739\end{array}$ & $\begin{array}{l}1.85 e^{-4^{* *}} \\
2.84 e^{-4^{* *}} \\
2.02 e^{-5^{* *}}\end{array}$ \\
\hline
\end{tabular}

In most cases, the value of the MSE obtained by the proposed method is lower than that of the methods for comparison (negative t-statistic values), with significance values much lower than 0.01 . It is thus demonstrated that, overall, the proposed method significantly outperforms the other methods proposed in the state-of-the-art literature. 


\section{Discussion and conclusions}

This article presented a hybrid recommender system that uses matrix factorization techniques. Such system integrates ratings, as well as user and item characteristics, in order to estimate the relationships that exist between such characteristics and offer educational resources that help students in their learning process in a virtual environment.

In order to conduct validations and make comparisons with other models proposed in the literature, a data set was created with a total of 400 registers (students $=56$, OERs $=152$, and ratings $=$ 400). In it, the learning style of each student was defined as a representative characteristic. Several metadata of the OERs were utilized, especially those related to the educational category. Therefore, the educational description by the author of the resource or the user who tagged it could be used to define the extent to which such resource could contribute to each learning style. Additionally, the ratings were classified into 5 different categories: overall rating, contribution to the learning process, design, content quality, and likelihood of recommending the resource to other users.

The proposed model $\left(M_{1}\right)$, using known information about items and users, was compared with three other models proposed in the literature: $M_{2}$, where user information is known; $M_{3}$, where item data is available; and $M_{4}$, where user and item information are unknown. The MSE was used to make the comparison; the lower the MSE, the better the recommendation model. Two experiments were conducted. In the first one, the data set was divided into 10 partitions by means of cross validation; in the second, the cold start problem of a new user was implemented to perform the validation.

The validation in this study enables the authors to conclude that the proposed recommendation system produces better results because it is not only based on users' ratings, it also considers other types of information, which can be implicit or explicit. The hypothesis that the more information the better the results is therefore confirmed.

In future studies, other demographic characteristics of user profiles could be taken into account, and the cost function could be changed to consider nonlinear structures hidden in the ratings.

Author Contributions: PARM: Conceptualization, Methodology, Software, Validation, Formal analysis, Investigation, Data Curation, Writing - Original Draft, Visualization, NDDM: Conceptualization, Methodology, Investigation, Writing - Review and Editing, DAOC: Conceptualization,Investigation JDMV: Conceptualization, Methodology, Software, Validation, Formal analysis, Writing - Review and Editing, Visualization

Funding: This research received no external funding

Conflicts of Interest: The authors declare no conflict of interest.

1. George, G.; Lal, A.M. Review of ontology-based recommender systems in e-learning. Computers $\mathcal{E}$ Education 2019, 142, 103642. doi:https:/ / doi.org/10.1016/j.compedu.2019.103642.

2. $\quad$ UNESCO. Guía Básica de Recursos Educativos Abiertos (REA), organizaci ed.; Vol. 6, 2015; p. 152.

3. MEN, M.d.E.N. Estrategia REDA, Ministerio de Educación Nacional, 2013.

4. Campo Saavedra, M.F.; Martínez Barrios, P.D.P.; Ruíz Rodgers, N.; Rendón Osorio, H.J. Recursos Educativos Digitales Abiertos COLOMBIA; 2012.

5. Duque, N.; Ovalle, D.; Moreno, J. Objetos de Aprendizaje, Repositorios y Federaciones... Conocimiento para Todos; 2014; p. 173.

6. Tabares, V.; Duque, N.; Rodríguez, P.; Moreno, J.; Ovalle, D. FROAC : Una Iniciativa Colombiana para la Integración de Repositorios de Objetos. Campus Virtuales 2015, IV , 108-117.

7. Rodríguez, P.; Heras, S.; Palanca, J.; Poveda, J.; Duque, N.; Vicente Julián. An educational recommender system based on argumentation theory. AI Communications 2017, 30, 19-36. doi:10.1016/j.cub.2015.09.073.

8. Kay, R.; Knaack, L.; Petrarca, D. Exploring teachers perceptions of Web-based learning tools. Interdisciplinary Journal of E-Learning and Learning Objects 2009, 5, 27-50.

9. Gordillo, A.; Barra, E.; Quemada, J. A Hybrid Recommendation Model for Learning Object Repositories. IEEE LATIN AMERICA TRANSACTIONS, 2017, 15, 462-473. doi:10.1002/ace. 
10. Identifying the goal, user model and conditions of recommender systems for formal and informal learning. Journal of Digital Information 2009, 10, 1-17.

11. Nabizadeh, A.H.; Gonçalves, D.; Gama, S.; Jorge, J.; Rafsanjani, H.N. Adaptive learning path recommender approach using auxiliary learning objects. Computers E Education 2020, 147, 103777. doi:https://doi.org/10.1016/j.compedu.2019.103777.

12. Khenissi, M.A.; Essalmi, F.; Jemni, M.; Kinshuk.; Graf, S.; Chen, N.S. Relationship between learning styles and genres of games. Computers $\mathcal{E}$ Education 2016, 101, 1 - 14. doi:https://doi.org/10.1016/j.compedu.2016.05.005.

13. Reategui, E.; Boff, E.; Campbell, J. Personalization in an interactive learning environment through a virtual character. Computers $\mathcal{E}$ Education 2008, 51, 530 - 544. doi:https://doi.org/10.1016/j.compedu.2007.05.018.

14. Aggarwal, C.C. Recommender systems: The Textbook; Number 1, 2016; pp. 1-518, [1202.1112]. doi:10.1007/978-3-319-29659-3.

15. Zhang, F. A Personalized Time-Sequence-Based Book Recommendation Algorithm for Digital Libraries. IEEE Access 2016, 4, 2714-2720. doi:10.1109/ACCESS.2016.2564997.

16. Liu, Y.; Qu, H.; Chen, W.; Mahmud, S.M.H. An Efficient Deep Learning Model to Infer User Demographic Information From Ratings. IEEE Access 2019, 7, 53125-53135. doi:10.1109/ACCESS.2019.2911720.

17. Kong, X.; Member, S. A Survey of Collaborative Filtering-Based Recommender Systems : From Traditional Methods to Hybrid Methods Based on Social Networks. IEEE Access 2018, 6, 64301-64320. doi:10.1109/ACCESS.2018.2877208.

18. Gu, Q.; Zhou, J.; Ding, C. Collaborative filtering: Weighted nonnegative matrix factorization incorporating user and item graphs. Proceedings of the 10th SIAM International Conference on Data Mining, SDM 2010 2010, pp. 199-210. doi:10.1137/1.9781611972801.18.

19. Koren, Y.; Bell, R.; Volinsky, C. Matrix Factorization Techniques for recommender systems. Computer 2009, $42,42-49$.

20. Rodriguez, P.; Tabares, V.; Ovalle, D.; Duque, N. Modelo multi-agente para recomendación híbrida de objetos de aprendizaje. Revista virtual Universidad Católica del Norte 2013, 40, 96-110.

21. Polatidis, N.; Georgiadis, C.K. A dynamic multi-level collaborative filtering method for improved recommendations. Computer Standards and Interfaces 2017, 51, 14-21. doi:10.1016/j.csi.2016.10.014.

22. Luo, X.; Zhou, M.; Xia, Y.; Zhu, Q. An efficient non-negative matrix-factorization-based approach to collaborative filtering for recommender systems. IEEE Transactions on Industrial Informatics 2014, 10, 1273-1284. doi:10.1109/TII.2014.2308433.

23. Alexandridis, G.; Siolas, G.; Stafylopatis, A. Enhancing social collaborative filtering through the application of non-negative matrix factorization and exponential random graph models. Data Mining and Knowledge Discovery 2017, 31, 1031-1059. doi:10.1007/s10618-017-0504-3.

24. Zhao, C.; Sun, S.; Han, L.; Peng, Q. Hybrid Matrix Factorization for Recommender Systems in Social Networks. Neural Network World 2016, 26, 559.

25. Zhang, S.; Wang, W.; Ford, J.; Makedon, F. Learning from incomplete ratings using non-negative matrix factorization. Proceedings of the 2006 SIAM international conference on data mining. SIAM, 2006, pp. 549-553.

26. Gurini, D.F.; Gasparetti, F.; Micarelli, A.; Sansonetti, G. Temporal people-to-people recommendation on social networks with sentiment-based matrix factorization. Future Generation Computer Systems 2018, $78,430-439$.

27. Wu, H.A.O.; Zhang, H.; He, P.; Zeng, C.; Zhang, Y.A.N. A Hybrid Approach to Service Recommendation Based on Network Representation Learning. IEEE Access 2019, 7, 60242-60254. doi:10.1109/ACCESS.2019.2913947.

28. Ocepek, U.; Bosnić, Z.; Šerbec, I.N.; Rugelj, J. Exploring the relation between learning style models and preferred multimedia types. Computers $\mathcal{E}$ Education 2013, 69, 343 - 355. doi:https://doi.org/10.1016/j.compedu.2013.07.029.

29. Fleming, N.; Baume, D. Learning Styles Again: VARKing up the right tree! Educational Developments 2006.

30. Felder, R.M.; Silverman, L.K.; others. Learning and teaching styles in engineering education. Engineering education 1988, 78, 674-681.

31. IEEE-LTSC. IEEE Standard for Learning Object Metadata Summary of Changes from to Final Draft * LOM Standard v1 . 0, 2002. 
418 32. Adomavicius, G.; Tuzhilin, A. Toward the Next Generation of Recommender Systems : A Survey of 419 the State-of-the-Art and Possible Extensions. IEEE TRANSACTIONS ON KNOWLEDGE AND DATA $420 \quad$ ENGINEERING 2005, 17, 734-749.

421 33. Ricci, F.; Rokach, L.; Shapira, B. Recommender System Handbook, springer n ed.; 2011; pp. 1-848. 422 doi:10.1007/978-0-387-85820-3. 